\title{
Tech-footprints for virtual medicine
}

\section{Dear Readers,}

The health care driven tools and solutions speed up the overall time for treatment as well as create better enduring experiences, which is the need of the hour, at the same time. It eventually raises the standards of the health care industry across the spectrum by understanding as well as responding to customers in a systematic and logical manner. Computational and information system that mines healthcare database with improve treatment cost-effectively, seeking opportunities for growth in industry regularly. Challenges and opportunities were counterbalance by artificial intelligence (AI), information technology, 3-D printing etc. in astounding ways to encourage healthy lifestyles. Besides that, these modern tech tools and solutions have the ability to create a wide range of AIenabled medical models, as well as develop and analyze critical data, and bear in mind, all-in-one integrated medicine for streamlined care.

According to recent studies, the tech advancements not only provides key insights, but also integrate modern channels too such as emails, social media and more patterns to foster better relationships. They are designed to reveal invaluable insights of digital medicine from the current trends in real time, which has the ability to empower the entire health care industry.

An unfavourable/ error occasion is characterized as damage caused by restorative administration instead of by the hidden sickness or state of the patient. Not all, but rather a sizable extent of unfriendly occasions is the after effect of mistakes. Various investigations have taken a gander at the extent of unfavourable occasions owing to medicinal mistake. Due to methodologic challenges, far less investigations center around the full scope of mistake - in particular, those that outcome in damage and those that open the patient to chance however don't result in damage.

AI-enabled technologies are making great strides regularly, reflected by increasing virtual medicine startups. The intelligent healthcare systems are contributing effectively due to tech footprints, spread in hospitals and health industries through advanced devices which penetrated the human care systems at a deeper level. These tools not only curate intelligent data and systematize analytic asset discovery, and governance, but also improve the delivery model. It can empower industry experts to deeply analyze/study/break/ understand text to extract metadata from the extensive content; which may or may not include integral concepts, as well as entities.
The decade groomed with the cloud know-how, upgraded the healthcare system more and making it easier than ever before. The entire practice of high-tech healthcare can be revolutionized by intelligent technologies making wide acceptability to reduce world vulnerability towards the intelligent lifestyle.

With kindest regards

\section{Dr. Ashutosh Tiwari \\ Editor-in-Chief \\ Advanced Materials Letters}

Keywords: Digital healthcare, virtual medicine, all-inone integrated care, intelligent lifestyle. 